\title{
A stereoscopic PIV system for the Princeton Superpipe
}

\author{
Department of Mechanical and Aerospace Engineering \\ Princeton University, Princeton, New Jersey, USA \\ ${ }^{*}$ Corresponding author: liuyangd@princeton.edu
}

L. Ding*, E. Limacher, I. E. Gunady, A. Piqué, M. Hultmark and A. J. Smits

\begin{abstract}
Herein, we describe the design and testing of a stereoscopic PIV system uniquely adapted for the high pressure environment of the Princeton Superpipe. The Superpipe is a recirculating pipe facility that utilizes compressed air as the working fluid to attain very high Reynolds numbers. Commercial piping is used as the pressure vessel to hold pressure up to 220 bars, and a test pipe is enclosed inside with a development length of 200 diameters that ensures a fully-developed condition at the test section. The highest achievable Reynolds number (based on the bulk velocity and the pipe diameter) is $35 \times 10^{6}$, corresponding to a maximum friction Reynolds number of $5 \times 10^{5}$.

The unprecedented range of Reynolds number has enabled a number of new insights in the behavior of high Reynolds number wall-bounded turbulence (Zagarola and Smits, 1998; Hultmark et al., 2013). However, past measurements in the Superpipe have been primarily restricted to single-component, one- or two-point statistics of fully-developed pipe flows. The present work aims to expand the capability of the Superpipe to study turbulent coherent structures and multi-point statistics by means of a new stereoscopic PIV system. The high pressure environment and the confined space inside the pressure vessel pose challenges to both imaging and seeding, the solutions to which will be discussed.
\end{abstract}

\section{Imaging}

The imaging system has been designed so that only mirrors are contained in the high-pressure environment, and all cameras are located external to the test section. This configuration offers a significant benefit - its performance is nearly unaffected when the refractive index of compressed air changes with pressure. More specifically, the object plane is chosen to be the one determined by the axes of the test pipe and the access port of the test section (see figure 1). A laser light sheet is delivered into the test pipe through the sight windows on the sealing flange and the internal test pipe. An optical relay assembly consisting of two nearaxis and two off-axis mirrors is mounted directly to the test pipe and used to relay particle images to cameras placed outside the pressure vessel through the flange sight window. As indicated by the simulated light rays in figure 1 , the mirrors on each side of the light sheet provide one view of the object plane, so the two pairs of mirrors constitute a stereoscopic system.

By means of optical ray tracing, the imaging system is optimized in terms of the following aspects: (i) the angle between the two views in stereoscopic imaging is $90^{\circ}$ to minimize the uncertainty in the azimuthal velocity component (Lawson and Wu, 1997); (ii) the field of view encompasses the region between the wall and the centerline of the pipe and spans at least one radius $(R=63.8 \mathrm{~mm})$ in the pipe axial direction; (iii) the chief ray emanating from the center of the field of view $(y=R / 2$ with $y$ being the wall-normal distance) is perpendicular to the sight window on the sealing flange (\#3 in figure 11) to minimize astigmatism. With the ray tracing analysis, we have also evaluated the images of point sources in the object plane. The image sizes across the field of view are smaller than $10 \mu \mathrm{m}$, as compared to the diffraction-limited spot size of 17 $\mu \mathrm{m}$ at the same magnification and $f$-number.

\section{Seeding}

The design of the flow seeding system must address the following challenges: (i) it must allow for the generation and injection of seeding particles at or near the desired test pressures; (ii) it must not foul the internal surfaces of the Superpipe, which is difficult to clean; and (iii) it must produce a narrow distribution of particle sizes with a mean diameter around $1 \mu \mathrm{m}$.

Our chosen design consists of a sealed, pressure-rated housing that serves as a liquid reservoir, with several Laskin nozzles submerged in the liquid. Compressed air at higher pressure is driven through the nozzles to generate an aerosol, which is then carried into the Superpipe through a connecting tube. We have 


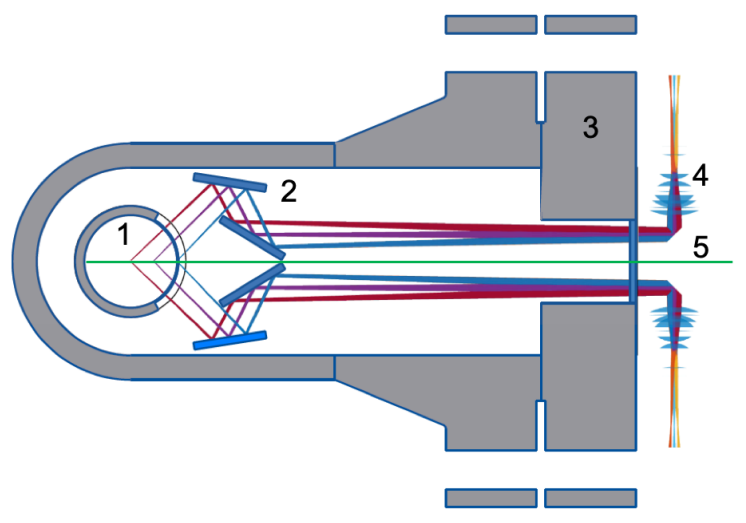

Figure 1: The imaging system shown in a crosssection of the access port, with simulated light rays indicating the imaging paths. 1: test pipe with a thin sight window. 2: mirrors (two near-axis ones and two off-axis ones). 3: sealing flange with a sight window. 4: lens group of a $135-\mathrm{mm}$ prime lens. 5: laser sheet.

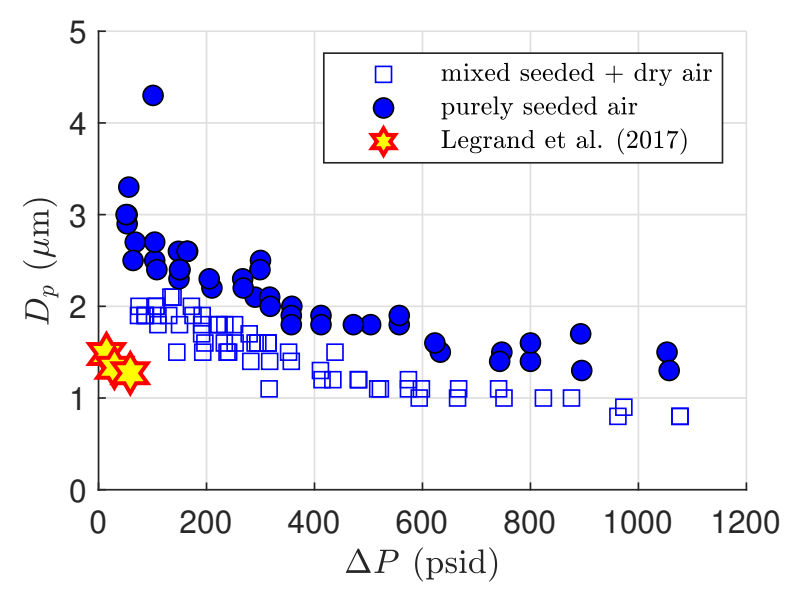

Figure 2: $D_{p}$ versus $\Delta P$ for all test cases run. Filled circles denote purely seeded cases; empty squares represent cases with dry air mixed with seeded air. Yellow-filled red stars are results from Legrand et al. (2017) with no dry air added.

opted for propylene glycol as the seeding liquid because it is inexpensive with a low vapor pressure - a desirable property keeping the surfaces of the test section free of liquid residue.

A Dantec PDA system was used to characterize the size distribution of droplets produced by the seeder. The modal (peak) diameter $D_{p}$ is plotted in figure 2 versus the pressure difference, $\Delta P=P_{1}-P_{2}$, where $P_{1}$ is the supply pressure, and $P_{2}$ is the housing pressure. These results are for a nozzle with two $0.51 \mathrm{~mm}-$ diameter holes; other configurations were also tested, but are omitted for brevity. In general, increasing the nozzle pressure difference tends to decrease droplet sizes, and adding dry air for a given configuration and $\Delta P$ tends to reduce the droplet size further, presumably due to evaporation. To confidently get a droplet size distribution with a modal diameter of $2 \mu \mathrm{m}$ or less, one should maintain a pressure difference across the nozzle of about 500 psid, if purely seeded air is desired. Mixing dry air is likely desirable to achieve seeding densities more appropriate to PIV. However, it bears noting that these results and the above suggestion are sensitive to other system design parameters that are not fully understood. Legrand et al. (2017), for example, managed to obtain smaller modal diameters than us at low pressure differences (yellow-filled red stars on figure 2).

\section{System Integration}

Having designed, assembled and tested various subsystems, we have moved on to begin testing their integration. We will present preliminary results showing acquired particle images in the test pipe at ambient pressure, and we will discuss recently addressed and ongoing challenges such as background reflection and droplet condensation.

\section{Acknowledgements}

This work is supported by ONR DURIP Grant No. N00014-19-1-2301 and ONR Grant No. N00014-17-12309. IEG was supported by the Department of Defense (DoD) through the National Defense Science and Engineering Graduate Fellowship (NDSEG) Program.

\section{References}

Hultmark M, Vallikivi M, Bailey SCC, and Smits A (2013) Logarithmic scaling of turbulence in smooth-and rough-wall pipe flow. Journal of Fluid Mechanics 728:376-395

Lawson N and Wu J (1997) Three-dimensional particle image velocimetry: error analysis of stereoscopic techniques. Measurement Science and Technology 8:894-900

Legrand M, Nogueira J, Rodriguez PA, Lecuona A, and Jimenez R (2017) Generation and droplet size distribution of tracer particles for PIV measurements in air, using propylene glycol/water solution. Experimental Thermal and Fluid Science 81:1-8

Zagarola MV and Smits AJ (1998) Mean-flow scaling of turbulent pipe flow. Journal of Fluid Mechanics 373:33-79 